\title{
Event-related potentials in a two-interval forced-choice detection task
}

\author{
SAMUEL SUTTON \\ New York State Psychiatric Institute, New York, New York \\ DANIEL S. RUCHKIN and ROBERT MUNSON \\ University of Maryland School of Medicine, Baltimore, Maryland \\ MITCHELL L. KIETZMAN \\ Queens College of CUNY, Flushing, New York \\ and New York State Psychiatric Institute, New York, New York \\ and \\ MURIEL HAMMER \\ New York State Psychiatric Institute, New York, New York
}

\begin{abstract}
In an attempt to elucidate the nature of the subject's strategy in a two-interval forcedchoice auditory detection task, event-related potentials were studied at two intensities which yielded mean accuracies of $82 \%$ and $98 \%$. Subjects reported the observation interval in which they judged the signal to be present and the confidence of the judgment. Principal components varimax analyses yielded four components: a CZ maximal P300, a Slow Wave, a slow negative shift, and a late negative component. The P300 amplitude findings suggest that different strategies are utilized for high-confidence and low-confidence detections. At high confidence, P300 amplitude is large for the observation interval in which the signal is presented, indicating a strategy involving serial independent detection. However, the P300 latency findings at high confidence suggest that absence of the signal in the first observation interval is nonetheless noted: P300 latency in response to signal presence is shorter for the second observation interval than for the first observation interval. At low confidence, P300 is small or absent for both observation intervals, indicating a deferred decision, presumably arrived at through comparison of the two percepts.
\end{abstract}

There are several studies in which the P300 component of the event-related potential has been investigated in relation to yes/no detection accuracy (Barrett, Halliday, Halliday, \& Rudolf, 1979; Campbell, Courchesne, Picton, \& K. Squires, 1979; Hillyard, K. Squires, Bauer, \& Lindsay, 1971; Ruchkin, Sutton, Kietzman, \& Silver, 1980; Wilkinson \& Seales, 1978). In general, the findings have been that $\mathrm{P} 300$ is larger the higher the detection accuracy (as manipulated by signal intensity within the psychometric range in these studies). P300 is also larger the higher the rated confidence of the decision (Kerkhof, 1982; K. Squires, Hillyard, \& Lindsay, 1973; K. Squires, N. Squires, \& Hillyard, 1975a; K. Squires, N. Squires, \& Hillyard, 1975b; Wilkinson \& Seales, 1978).

In contrast to yes/no (single interval) detection,

We are indebted to Phillip Ramsey for advising on the statistical analysis, and to Janet Camp, W. Crawford Clark, David Friedman, Lois Putnam, Jacob Rutschmann, Nancy Squires, Michael Terman, and Walter Ritter for commenting on various drafts of this manuscript. This research was supported in part by a USPHS grant, NINCDS 11199 . Correspondence may be directed to Samuel Sutton, Chief, Department of Psychophysiology, New York State Psychiatric Institute, 722 West 168th Street, New York, New York 10032. for multiple-interval forced-choice detection, data on event-related potential correlates are quite sparse. In multiple-interval forced-choice procedures, the signal is present in every trial, but occurs in only one of the observation intervals. Picton, Campbell, Baribeau-Braun, and Proulx (1978, p. 438) reported that, in a four-interval forced-choice detection paradigm, "late positive waves occurred in response to informative stimuli, particularly the actual signal stimuli. The late positive component was maximally recorded from the parietal region." However, only illustrative data were presented and it was not possible from these data to evaluate the exact nature of their findings.

There are special and subtle issues which arise in multiple-interval forced-choice procedures, and it is possible that event-related potential data can cast some light on these issues. Treisman and Leshowitz (1969) argued that, at least for one type of twointerval forced-choice procedure, the data could best be interpreted by assuming that the subject uses a serial independent detection strategy. In other words, the subject makes a decision for each observation interval as to whether the perception in that interval exceeds the noise criterion, and then reports the observation interval for which this criterion is ex- 
ceeded. These authors were contrasting this strategy with a differencing strategy (attributed to Tanner and Swets) which assumes that the subject is taking differences between the percepts in the multiple observation intervals. For example, consider the situation in which the subject is trying to decide whether a signal of very low intensity was presented in the first or the second observation interval of a two-interval forced-choice procedure. The differencing strategy would imply that the subject decides whether interval a yields a more signal-like perception than interval $b$ or, converseley, interval $b$ yields a more signallike perception than interval a. The interval in which the more signal-like perception is obtained is the one that is reported as the interval in which the signal is present.

In an attempt to shed light on what the subject is doing in a multiple-interval forced-choice procedure, we used one characteristic property of the P300 component of the event-related potential, namely that the P300 component is sensitive to the event in the trial from which critical information is extracted.

The generalization that $\mathbf{P} 300$ is largest in relation to events from which critical information is extracted is implicit in much of the P300 literature. However, in relatively few experiments has the location in the trial at which maximum information is available been manipulated symmetrically. Sutton, Tueting, Zubin, and John (1967) manipulated the relative informational properties of two different events in a trial by having the subject operate according to different instructions in different blocks of trials. Under one set of instructions, the subject predicted whether single or double clicks would occur, and correctness with respect to this criterion determined payoff. The fact that clicks were sometimes loud and sometimes soft was irrelevant for the single-double condition. In other blocks of trials, the subject was instructed to predict whether clicks would be loud or soft, and correctness with respect to this criterion determined payoff. The fact that clicks were sometimes single and sometimes double was irrelevant for the loudsoft condition. The P300 findings clearly reflected the fact that critical information was at different locations in the trial for the two instructional conditions (Sutton et al., 1967, Figure 2). Under the single-double instruction, the first event (a click that was always present) marked time but was not otherwise informative. The second event delivered the critical information. The second event had a large P300 regardless of whether or not a click was present at that time. In contrast, under the loud-soft instruction, the second click was informationally completely redundant, and under this instructional condition P300 to the second event was completely absent, regardless of presence or absence of the click at that time. For the first click, P300 was clearly larger under the loud-soft instruction than under the single-double instruction. This reflected the fact that for the loud-soft instruction the critical information was available at the first event. That P300 to the first click was small rather than absent under the singledouble instruction probably reflected the fact that for this condition the first click served a time-marking function.

Other experiments which manipulated the location of information in the trial used letters or words. Shelburne (1972) reported that when all the information was present in the last letter of a trigram, P300 was largest for the last letter, whereas when all the information was present in the first letter, P300 was largest for the first letter. However, Friedman, Simson, Ritter, and Rapin (1975) did not obtain comparable results for words in sentences. Here, P300 was always largest for the last word regardless of the location of the critical information. They interpreted their findings as reflecting the inherent syntactical structure of sentences which require completion even when no additional information is provided by the final word.

That events which contain critical information produce a larger $\mathrm{P} 300$ because more information is extracted from these events is illustrated by Johnson and Donchin (1982). The relative frequency of two stimuli was shifted unpredictably and, in one experimental condition, the subject was asked to report the point at which this shift was perceived. In this condition, P300 amplitude increased monotonically as the point of the report of shift was approached, that is, as the subject became increasingly convinced that a probability shift had occurred. This finding was not obtained for a condition in which the subject had not been told that probability shifts would occur. Findings leading to the same conclusion were reported by Ritter and Vaughan (1969). In a vigilance task, detected rare signals yielded a large $P 300$, whereas those rare signals that were not detected yielded a small, or no, P300; that is, for undetected rare signals, a waveform was obtained which was indistinguishable from the waveform obtained for the frequent nonsignals. Again, the conclusion is that $\mathbf{P} 300$ reflects the extraction of information from the signal rather than merely the presence of the signal.

Given the sensitivity of $\mathrm{P} 300$ to the point in the trial at which critical information is extracted, we reasoned that, in a two-interval forced-choice paradigm, the point in the trial at which the largest P300 is obtained might indicate the nature of the subject's detection strategy. This notion can be clarified by listing the implications of a few of the possible different findings:

(1) P300 is largest for the first observation interval whether the signal is presented in the first or second observation interval. Such a finding would be strong support for the notion that, since the information in the two observation intervals is logically redundant, 
the subject extracts all the necessary information in the first observation interval.

(2) P300 is largest for the second observation interval whether the signal is presented in the first or second observation interval. Such a finding would be strong support for the notion that the subject is engaged in a comparison operation such as differencing. Since a comparison cannot be made until the information provided by the second observation interval has been extracted, the largest P300 would be obtained in response to the information in this interval.

(3) P300 is small or absent for both observation intervals. This would be interpreted as weak support for a comparison strategy, since the absence of a P300 would suggest that neither observation interval provided sufficient information. Such a finding would suggest that the subject is utilizing the information in both observation intervals (perhaps making a comparison) but reserving decision until a report must be made.

(4) P300 is largest in response to the observation interval in which the signal is presented and small or absent in response to the other observation interval. Such a finding would indicate (1) that in a twointerval forced-choice detection task the subject makes serial independent detections, and (2) that at least in the two-interval forced-choice detection paradigm, the subject extracts most of the information from signal presence and relatively little information from signal absence.

In our experimental design, both observation intervals were marked by a clearly suprathreshold visual stimulus which had the same duration as the auditory signal that was to be detected. Such cuing of the observation intervals leaves no uncertainty as to when to search for the signal (Egan, Greenberg, \& Schulman, 1964), and it thereby reduces trial-totrial latency jitter of endogenous components. This is particularly important for the measurement of average $\mathbf{P} 300$ amplitude in response to signal absence (K. Squires et al., 1975a).

Our experimental design was based on the notion that P300 findings might shed some light on decision strategy in a two-interval forced-choice detection task. While we expected that components in addition to P300 would be obtained, explicit hypotheses about the findings for other components were not part of the original design. Rather, for one of these other components, namely parietal Slow Wave, which is also obtained in detection paradigms (Ruchkin, Sutton, Kietzman, \& Silver, 1980), it was hoped that the findings of the present study would provide further clues as to its functional role.

\section{METHOD}

Subjecta

There were 13 subjects, 9 male and 4 female, with a median age of 24 years (range, 19-42). Twelve were paid volunteers and the 13th was one of the experimenters (D.S.R.).

\section{Procedure}

The subject, wearing a set of headphones, was seated in an IAC sound-treated chamber. The signals were $1,000-\mathrm{Hz}$ tones presented to the right ear only. They had a 5-msec rise and fall time and their total duration was 50 msec. They were presented against a continuous background white noise at an intensity of $65 \mathrm{~dB}$ re .0002 dynes/ $\mathrm{cm}^{2}$ to mask any residual external or internal noise, so that, in fact, the tone threshold was being measured above the background noise level. In different blocks, we used two intensity levels: one at about the $80 \%$ accuracy level (Lo) and one at the $95 \%-99 \%$ accuracy level (Hi). The intensity levels were set independently for each subject based on pilot testing. Across subjects, the range of attenuations relative to an arbitrary level was 26 to $30 \mathrm{~dB}$ for the Lo stimuli and 22 to $25 \mathrm{~dB}$ for the $\mathrm{Hi}$ stimuli. The obtained accuracy across subjects averaged $82 \%$ for the Lo condition and $98 \%$ for the $\mathrm{Hi}$ condition. Accuracy did not differ significantly $(p>.05)$ as a function of the observation interval in which the signal was presented at either $\mathrm{Hi}$ or Lo intensity.

Each block consisted of 75 trials. There were four blocks at each intensity. Lo and Hi blocks were alternated, and whether the first block of the experiment was $\mathrm{Lo}$ or $\mathrm{Hi}$ was counterbalanced across subjects.

The trial began when the subject fixated a red LED and put a finger on the start button. After a short random interval, a s0-msec light flash (green LED) was presented. This first green light flash was a warning to prepare the subject for the occurrence of the first observation interval. Seven hundred milliseconds later, a second 50 -msec green flash occurred which defined the first observation interval (OI 1). If the auditory signal was to be presented in that interval, its occurrence and duration corresponded to the occurrence and duration of the green flash. In order to allow sufficient time for recording the event-related potential, the second observation interval did not begin until 800 msec after the onset of the previous green flash. A third 50-msec green flash then occurred, and this defined the second observation interval (OI 2). As before, if the auditory signal was to be presented in that interval, it occurred simultaneously with that green flash.

Signals were presented in one or the other observation interval. A single Bernoulli sequence determined the observation interval in which the signal was presented. There were no trials in which signals were presented in both intervals or in neither interval. The subject did not report the decision until the red fixation light was turned off, which was at least $2 \mathrm{sec}$ after the onset of the second observation interval. The decision was reported by pressing one of four buttons indicating the interval in which the signal was perceived and the degree of rated confidence (high or low).

For the balance of this paper, the term "observation interval" refers to the period ( $50 \mathrm{msec}$ ) defined by the second cuing flash (OI 1) or the third cuing flash (OI 2). The term "recording epoch" refers to the segment of data being analyzed. Thus, the warning interval recording epoch begins with the onset of the first green flash and ends at the onset of the second green flash $(700 \mathrm{msec})$. The OI 1 recording epoch begins with the onset of the second green flash and ends at the onset of the third green flash $(800 \mathrm{msec})$. The OI 2 recording epoch begins with the onset of the third green flash and ends 1,200 msec later. A longer duration is used for the second recording epoch simply because it is available.

\section{EWG Recordling}

Event-related potentials (ERPs) were recorded from midline occipital, parietal, central, frontal, and prefrontal scalp, all referred to linked earlobes. The ground electrode was on the left wrist. Eye movement artifact was monitored by a pair of electrodes, one above the inner canthus and one below the outer canthus of the left eye. Silver/silver chloride electrodes were used. Prior to application of the electrodes, the skin was cleaned with alcohol and gently abraded and the epidermis was punctured 
with a sterile lancet. Electrode resistance was below $1.8 \mathrm{~K} \Omega$ at all loci. PAR Model 113 amplifiers, modified to increase input resistance, were used. The amplifiers were set to a gain of 10,000, an upper cutoff $(-3 \mathrm{~dB})$ frequency of $30 \mathrm{~Hz}$, and an ac coupling time constant of $5.3 \mathrm{sec}$. A digital computer controlled the sequence, selection, and timing of events in each trial and digitized and monitored the data on-line (sampling rate of $100 \mathrm{~Hz}$ ). Data were stored on digital magnetic tape for off-line analysis. Prior to further computations, the root mean square amplitude of the electrooculogram (EOG) was computed for each trial. If the root mean square amplitude exceeded $15 \mu \mathrm{V}$, the trial was not used.

\section{Data Analysis}

For each subject, average ERPs were computed separately for each cell of the interaction of (1) signal location, (2) confidence level, (3) signal intensity, and (4) whether or not the subject correctly detected the signal. Since subjects were operating at relatively high levels of accuracy, there were an insufficient number of trials for systematic analysis of the incorrect responses (false alarms and misses), and these data are not presented.

In the analysis of the data, principal components varimax analysis (PCVA) was used. A detailed discussion of our implementation of PCVA is presented in Ruchkin, Sutton, and Stega (1980). Our rationale for using PCVA is that the method may provide estimates of component amplitudes that are relatively free of temporal and spatial overlap which could confound baseline-to-peak measures. The obtained basis waveforms (usually referred to in the psychological literature as loading functions) are viewed as candidates for the waveshapes of separate "underlying" components. It should be noted that the conviction that a given basis waveform corresponds to an underlying component does not arise from a particular PCVA or a particular experiment but, rather, rests on the convergence of several lines of evidence from many experiments. The weighting coefficients (usually referred to in the psychological literature as factor scores) associated with each basis waveform provide measures of the contribution of the basis waveform to the amplitudes of the set of data waves used in the analysis, and thus represent estimates of the amplitude of a given component by itself. Weighting coefficients obtained from the PCVAs are converted to microvolt units by multiplying the weighting coefficients by the peak values of the corresponding basis waveform (which is in microvolts). Conversion to microvolts has a dual advantage. It permits one to view the obtained results in a more familiar unit, and it also permits comparisons of findings for the same component across PCVAs.

Separate cross-products matrix PCVAs were calculated for each of the three epochs: the warning-interval recording epoch $(700 \mathrm{msec})$; the first observation-interval (OI 1) recording epoch $(800 \mathrm{msec}$ ); and the second observation-interval (OI 2$)$ recording epoch $(1,200 \mathrm{msec})$. For each PCVA, there were 520 average waveforms ( 2 signal locations $\times 2$ confidence levels $\times 2$ intensities $\times$ 5 electrode loci $\times 13$ subjects). In addition, in order to gain further insight into the identity of some of the components, a supplementary PCVA was computed for a long recording epoch $(1,800 \mathrm{msec})$ from $180 \mathrm{msec}$ before the onset of the OI 1 recording epoch to $820 \mathrm{msec}$ after the onset of the OI 2 recording epoch.

The PCVAs indicated that the overlap among components was too great to permit meaningful baseline-to-peak amplitude measures. The analysis and presentation of the data therefore is based almost exclusively on the PCVA-derived weighting coefficients for each component.

Because of the relatively large number of statistical tests, $p=.01$ was used as a cutoff level of confidence (more exactly, $\mathrm{p}<.0145$ ). Since repeated measures designs may entail inflated degrees of freedom (Jennings \& Wood, 1976), for analyses of variance (ANOVAs) that included all five electrodes, the GeisserGreenhouse correction was used. Where such an inflation occurs, degrees of freedom were reduced to 1 and $12(F=9.33, p=.01)$. For ANOVAs at single electrodes, degrees of freedom are always 1 and 12 , so the p levels are exact.

\section{RESULTS AND DISCUSSION}

Conventional averages. Figure 1 displays waveforms over the total recording epoch at all scalp electrodes averaged across 13 subjects for trials in which the subjects correctly detected at high confidence the observation interval in which the signal was presented. As can be seen in Figure 1, for high-confidence detections there are clear differences as a function of the observation interval in which the signal was presented. Note particularly averages at $\mathrm{Cz}$ and $\mathrm{Pz}$. When the signal was presented in $\mathrm{OI} 1$, what appears to be a mix of P300 and Slow Wave can be seen in that recording epoch, whereas the late positivities are small or absent in the OI 2 recording epoch. In contrast, when the signal was presented in OI 2, the late positivities are large in that recording epoch, but small or absent in the OI 1 recording epoch.

For low-confidence detections (Figure 2), one cannot discern a clear pattern of findings.

P300 latency was examined at Cz, since the PCVAs (see below) yield a clearly $\mathrm{Cz}$ maximal $\mathrm{P} 300$ component. Latency was examined for the high-confidence averages only, since $P 300$ could not be reliably identified in the low-confidence averages. Latencies were as follows: Hi-intensity OI 1 recording epoch, $389 \mathrm{msec}$; Hi-intensity OI 2 recording epoch, $277 \mathrm{msec}$; Lo-intensity OI 1 recording epoch, $429 \mathrm{msec}$; Lo-intensity OI 2 recording epoch, $341 \mathrm{msec}$. Pooled over intensities, P300 latency was $100 \mathrm{msec}$ shorter in the OI 2 recording epoch than in the OI 1 recording epoch $(F=11.90, p=.005)$. Pooled over recording epochs, P300 latency was 52 msec shorter in the $\mathrm{Hi}$-intensity condition than in the Lo-intensity condi-

\section{HIGH CONFIDENCE}

HI INTENSITY

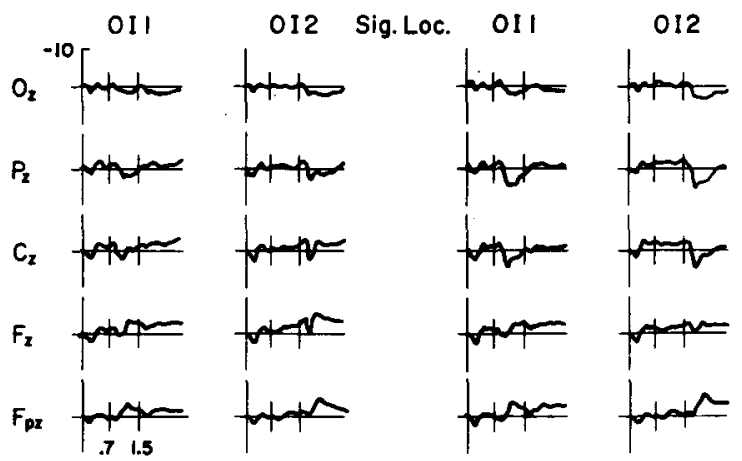

Figure 1. Total recording epoch average waveforms across 13 subjects at all scalp locations for high-confidence detections. The left pair of columns are for Hi-intensity signals; the right pair of columns are for Lo-intensity signals. Within each pair of columns, the left-colomn avernges are for signals located (Sig. Loc.) in OI 1 asd the right-column averajes are for algnals located in OI 2. In each avernge waveform, the two vertical lines separnte the three segments of the total recording epoch: warning interval recording epoch, OI 1 recording epoch, 012 recordling epoch. 


\section{LOW CONFIDENCE}

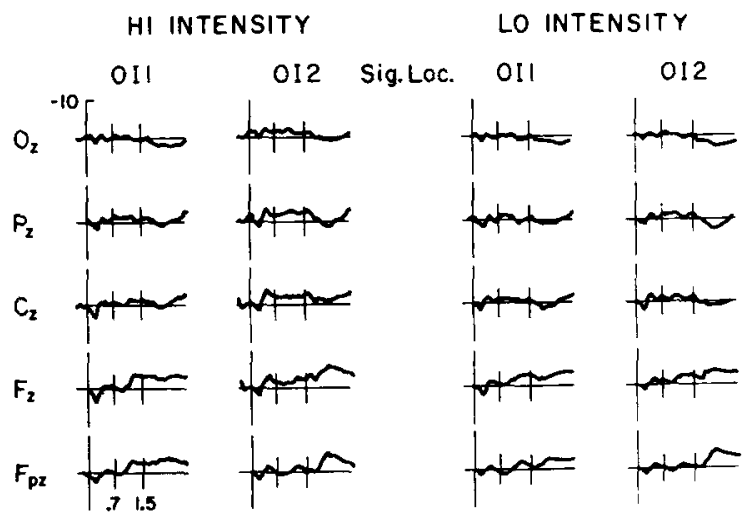

Figure 2. Average waveforms for low-confidence detections for the same categories as in Figure 1.

tion $(F=33.40, p=.00009)$. The signal location $x$ signal intensity interaction was not statistically significant.

Warning-interval recording epoch. The PCVA of the warning interval recording epoch yielded two components, a P220 which appeared largest at Pz (main effect of electrodes, $\mathrm{F}=6.69, \mathrm{p}<.05$ ) and $\mathrm{a}$ negative component, at the end of the epoch, which appeared largest at $\mathbf{C z}$ and $\mathrm{Fz}$ (main effect of electrodes, $F=2.21, p>.05$ ). None of the comparisons as a function of experimental conditions reached the .01 cutoff for statistical significance for either component.

OI 1 and 012 recording epochs. The PCVA of the OI 1 recording epoch yielded three components: a
P300 component, a Slow-Wave component, and a slow-negative-shift component. The PCVA of the OI 2 recording epoch yielded four components: a P300 component, a Slow-Wave component, a slownegative-shift component, and a late negative component. The separate PCVAs for the OI 1 recording epoch and the OI 2 epoch are presented together for each component. Following the results for each component, the discussion of findings for that component is presented. The bearing of the findings on the question of whether, in a two-interval forcedchoice paradigm, the subject is engaged in a serial independent detection strategy or in some kind of comparison strategy is discussed at the end of this paper.

For each component obtained in the PCVAs, complex interactions that are difficult to interpret were often found between electrode site and experimental variables. ANOVAs across electrodes were therefore used only to test the statistical significance of electrode distribution. Comparisons in relation to experimental variables are based on ANOVAs at whatever electrode generally yielded maximum amplitude for each component. For Slow Wave, however, since there is some evidence that positive amplitude at $\mathrm{Pz}$ and negative amplitude at Fz may represent different components (Fitzgerald \& Picton, 1981), separate ANOVAs were computed for the $\mathrm{Pz}$ and $\mathrm{Fz}$ data.

Table 1 shows $F$ and $p$ values for components at single electrodes. When comparisons reach the $.01 \mathrm{p}$ level, they are simply described in text; when they are between .01 and .05 , they are referred to in text as trends. $F$ and $p$ values are given in text only for results of tests that do not appear in Table 1.

Table 1

$F$ and p Values as a Function of Experimental Conditions for Components at Single Electrodes

\begin{tabular}{|c|c|c|c|c|c|c|c|}
\hline & \multirow[b]{2}{*}{$\begin{array}{c}\text { Recording } \\
\text { Epoch }\end{array}$} & \multicolumn{2}{|c|}{$\mathrm{P3} 00$ at $\mathrm{Cz}$} & \multicolumn{2}{|c|}{ Slow Wave at $\mathbf{P z}$} & \multicolumn{2}{|c|}{ Slow Wave at Fz } \\
\hline & & $\mathbf{F}$ & $\mathrm{p}$ & $\mathbf{F}$ & $\mathrm{p}$ & $\mathrm{F}$ & p \\
\hline Location & $\begin{array}{l}\text { OI } 1 \\
\text { OI } 2\end{array}$ & $\begin{array}{l}10.86 \\
20.70\end{array}$ & $\begin{array}{l}.0064 \\
.00067\end{array}$ & $\begin{array}{l}12.25 \\
13.77\end{array}$ & $\begin{array}{l}.0044 \\
.0029\end{array}$ & $\begin{array}{l}15.87 \\
10.20\end{array}$ & $\begin{array}{l}.0018 \\
.0077\end{array}$ \\
\hline Confidence & $\begin{array}{l}\text { OI } 1 \\
\text { OI } 2\end{array}$ & $\begin{array}{l}25.11 \\
11.80\end{array}$ & $\begin{array}{l}.0003 \\
.0049\end{array}$ & $\begin{array}{r}26.94 \\
3.64\end{array}$ & $\begin{array}{l}.00023 \\
.081\end{array}$ & $\begin{array}{r}.03 \\
2.57\end{array}$ & $\begin{array}{l}.87 \\
.13\end{array}$ \\
\hline Intensity & $\begin{array}{l}\text { OI } 1 \\
\text { OI } 2\end{array}$ & $\begin{array}{l}.01 \\
.01\end{array}$ & $\begin{array}{l}.92 \\
.91\end{array}$ & $\begin{array}{l}5.79 \\
5.89\end{array}$ & $\begin{array}{l}.033 \\
.032\end{array}$ & $\begin{array}{r}3.67 \\
10.40\end{array}$ & $\begin{array}{l}.080 \\
.0073\end{array}$ \\
\hline Location $\times$ Confidence & $\begin{array}{l}\text { OI } 1 \\
\text { OI } 2\end{array}$ & $\begin{array}{l}15.02 \\
23.90\end{array}$ & $\begin{array}{l}.0022 \\
.00037\end{array}$ & $\begin{array}{r}5.73 \\
20.92\end{array}$ & $\begin{array}{l}.034 \\
.00064\end{array}$ & $\begin{array}{l}.02 \\
.63\end{array}$ & $\begin{array}{l}.91 \\
.44\end{array}$ \\
\hline Location $x$ Intensity & $\begin{array}{l}\text { OI } 1 \\
\text { OI } 2\end{array}$ & $\begin{array}{r}.89 \\
8.37\end{array}$ & $\begin{array}{l}.36 \\
.014\end{array}$ & $\begin{array}{r}7.42 \\
11.73\end{array}$ & $\begin{array}{l}.019 \\
.005\end{array}$ & $\begin{array}{r}.44 \\
6.93\end{array}$ & $\begin{array}{l}.52 \\
.022\end{array}$ \\
\hline Confidence $x$ Intensity & $\begin{array}{l}\text { OI } 1 \\
\text { OI } 2\end{array}$ & $\begin{array}{r}.12 \\
4.04\end{array}$ & $\begin{array}{l}.74 \\
.067\end{array}$ & $\begin{array}{r}.01 \\
6.19\end{array}$ & $\begin{array}{l}.91 \\
.029\end{array}$ & $\begin{array}{r}.19 \\
3.58\end{array}$ & $\begin{array}{l}.67 \\
.083\end{array}$ \\
\hline Location $\times$ Confidence $X$ Intensity & $\begin{array}{l}\text { OI } 1 \\
\text { OI } 2\end{array}$ & $\begin{array}{r}1.34 \\
.86\end{array}$ & $\begin{array}{l}.27 \\
.37\end{array}$ & $\begin{array}{l}10.47 \\
29.78\end{array}$ & $\begin{array}{l}.0071 \\
.00015\end{array}$ & $\begin{array}{l}.41 \\
.18\end{array}$ & $\begin{array}{r}.53 \\
.68 \\
\end{array}$ \\
\hline
\end{tabular}




\section{P300 Results}

As can be seen in Figure 3, a P300 component was obtained in both OI 1 and OI 2 recording epochs. The basis waveforms are shown in the upper portion of the panel for each recording epoch. In the raw average waveforms, the $\mathrm{P} 300$ component can be best visualized by inspecting the $\mathrm{Cz}$ electrode for highconfidence detections (Figure 1). Note that in both
Figure 1 and Figure 3 the peak latency of P300 in the OI 2 recording epoch is earlier than in the OI 1 recording epoch. The electrode distribution by experimental condition is presented in the lower portion of the panel for each recording epoch (Figure 3). The main effect of electrodes is not statistically significant for either recording epoch (OI $1, F=2.09$, $\mathrm{p}=.097$; OI $2, \mathrm{~F}=2.01, \mathrm{p}=.11$ ). However, note that

\section{0}

\section{OII EPOCH}
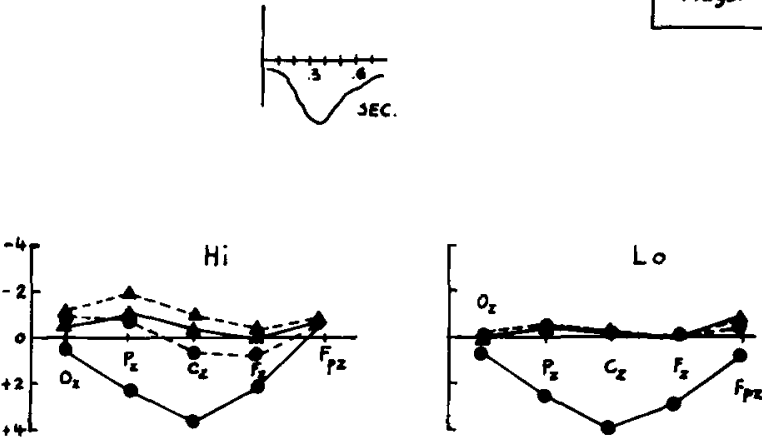

\section{OI 2 EPOCH}
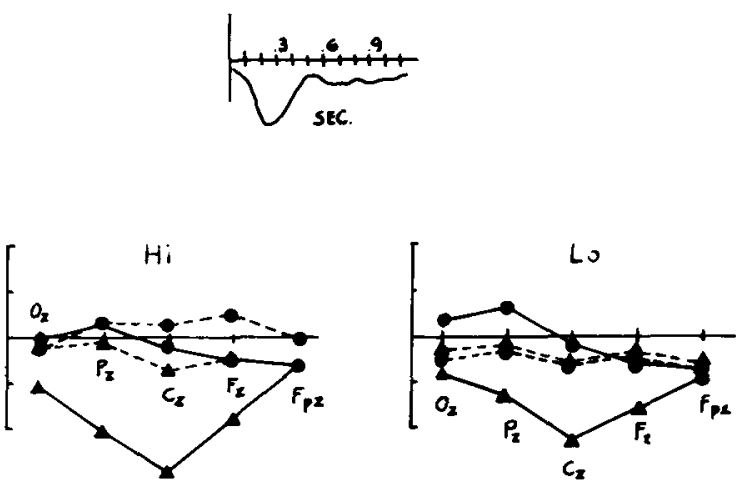

Figure 3. The P300 component derived from separate PCVAs of the OI 1 (upper panel) and OI 2 (lower panel) recording epochs. For each recording epoch, the basis waveform is shown above, and the distribution of amplitude (in microvolts) as a function of electrodes and experimental conditions is shown below. "Cert." refers to high-confidence detections; "Mayb." refers to low-confidence detections. 
in each set of conditions in Figure 3 (OI $1 \mathrm{Hi}$ and Lo, OI $2 \mathrm{Hi}$ and Lo), P300 amplitude is small and relatively flat across electrodes in three of the four curves, whereas in the only curve in each set of conditions in which P300 is large, the largest amplitude is found at $\mathrm{Cz}$.

$F$ and $p$ values for ANOVA comparisons for $P 300$ at $\mathrm{Cz}$ are shown in the first column of Table 1 . Plots of means across subjects for $\mathrm{P} 300$ at $\mathrm{Cz}$ are shown in Figure 4, with main effects in the upper half and two variable interactions in the lower half.

For both recording epochs, P300 is larger for signal present than for signal absent ("LOC"). Thus, in the OI 1 recording epoch, P300 is larger when the signal is present in OI $1(2.0 \mu \mathrm{V})$ than when the signal is absent in OI $1(-.5 \mu \mathrm{V})$. Correspondingly, in the OI 2 recording epoch, P300 is larger when the signal is present in OI $2(3.2 \mu \mathrm{V})$ than when it is absent in OI $2(.3 \mu \mathrm{V})$. P300 is also larger for high confidence ( $C$ for certain) than for low confidence $(M$ for maybe) in both recording epochs ("CONF").

P300 is largest at high confidence when the signal is present (OI $1,3.8 \mu \mathrm{V} ;$ OI $2,5.1 \mu \mathrm{V})$, and relatively small for all other conditions of the location $\times$ confidence interaction (" $\mathrm{LOC} \times \mathrm{CONF}$ ").

In the OI 2 recording epoch, the difference between P300 obtained in response to signal presence and P300 obtained in response to signal absence is greater at $\mathrm{Hi}$ intensity than at Lo intensity ("LOC $\times$ INT" for OI 2).

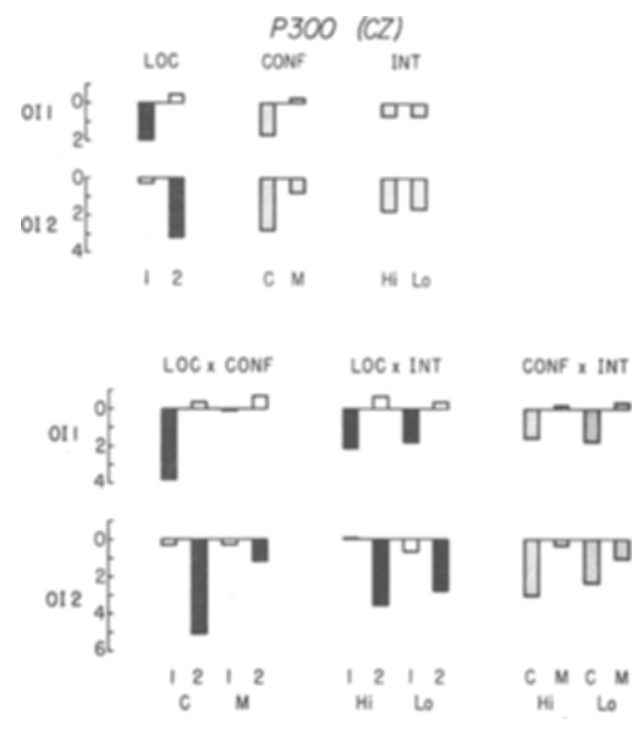

Figure 4. Across-subject means for $\mathrm{P} 300$ at $\mathrm{Cz}$ as a function of experimental conditions for OI 1 and $O I 2$ recording epochs. 1 and 2 = signal location; $C$ (certain) = high-confidence detections; $M$ (maybe) = low-confidence detections; $\mathbf{H i}=$ high-intensity condition; Lo = low-intensity condition. Black bars are categories in which the signal is present; open bars are categories in which the signal is absent; gray bars are categories which average across signal presence and signal absence. Note that for each pair of plots (OI 1 and 012 recording epochs), bars in the same vertical column represent the same set of trials.
The three-variable interaction for $\mathrm{P} 300$ at $\mathrm{Cz}$ was not statistically significant for either the OI 1 or OI 2 recording epoch.

Finally, for P300 at $\mathrm{Cz}$, there was no statistically significant difference in amplitude between the OI 1 recording epoch and the OI 2 recording epoch $(\mathrm{F}=4.20, \mathrm{p}=.063)$.

\section{P300 Discussion}

Signal location, signal presence vs. signal absence, and equivocation (unresolved uncertainty) are discussed below as separate issues, although they are intimately linked in the findings.

P300 and signal location. In the present design, P300 appears to be primarily governed by detection of the presence of the signal (Figure 4). This is consistent with the direction of findings reported by Picton et al. (1978). The findings are not consistent with the Friedman et al. (1975) amplitude findings in which P300 to the last event in a trial is largest regardless of the information content of the last event. The current findings are more like Shelburne's (1972). In our data, P300 is largest in response to the high-confidence detection of the presence of the signal whether this detection occurs at the beginning or the end of the trial. It may be that well-rehearsed sentences (used by Friedman et al.) constitute an intrinsic syntactic unit, while the two observation intervals in a two-interval forced-choice trial constitute a less integral unit. Rather, the two intervals represent two points in time at which information may be extracted. Similarly, in the Shelburne study, while the three letters of a word constituted an intrinsic unit, the three letters of a nonsense trigram did not. The occurrence of $Z, Q$, or $X$ as the first letter permitted an immediate decision that the trigram would not form a word.

The form of the interaction between signal location and rated confidence sheds further light on the relationship of both of these factors to the extraction of information in a two-interval forced-choice paradigm. P300 is large for high-confidence detections of signal presence and relatively small for the other three permutations ("LOC $\times$ CONF,"' Figure 4). In other words, neither signal presence nor high confidence by itself yields a large or intermediate amplitude for P300. These findings suggest that in order for maximum information to be extracted, not only must the signal be present, but it must be detected at high confidence. The relatively small amplitude of P300 for low-confidence detections whether or not the signal is present reinforces the conclusion that signal presence alone does not yield a large amplitude for $P 300$.

It might be remembered that Friedman et al. (1975) found that, while P300 amplitude was sensitive to syntactic closure regardless of the location of the information, P300 latency was sensitive to the location of the information regardless of syntactic 
closure. In our experiment, the P300 amplitude data suggest that for high-confidence detections, maximal information is extracted in the recording epoch corresponding to the observation interval in which the signal is presented. Yet there is evidence that the subject does not ignore the informational properties of signal absence in OI 1 , and this may facilitate information extraction in response to the presence of the signal in OI 2. For high-confidence detections, the latency of P300 for signals presented in the second observation interval is $100 \mathrm{msec}$ earlier than the latency of P300 for signals presented in the first observation interval. The earlier latency of P300 when the signal is presented in the second observation interval suggests that although only the presence of the signal elicits a decision, signal absence in the first observation interval has nonetheless been noted. Any registration of the absence of the signal in the first observation interval would facilitate more rapid extraction of the information that the signal is present in the second observation interval.

Emmerich, Gray, Watson, and Tanis (1972) have reported behavioral evidence that response latency in a detection task is correlated with the degree of rated confidence: latency is shorter, the greater the rated confidence. Kerkhof (1982) and K. Squires et al. (1973) have reported that P300 latency is shorter, the greater the rated confidence. Taken together, these findings suggest that shorter P300 latency when the signal is detected in the second observation interval reflects a greater assurance in the subject's rating of high confidence because the information that the signal was absent in the first observation interval has also been processed.

Another way of describing the contrast between signals detected in OI 1 and signals detected in OI 2 is to note that the subjective probability of signals in OI 1 is likely to correspond to the objective probability of .5 , whereas, since signal absence in OI 1 might be utilized as a source of information, the subjective probability of signals in OI 2 could be higher than .5. However, while such a formulation is consistent with the latency findings, it is not borne out by the amplitude findings. For P300 latency, we found a significant difference between signals detected in OI 1 and signals detected in OI 2 . Here the direction is what one would predict, shorter for higher probability (Duncan-Johnson, 1981). For P300 amplitude, which has been found to be inversely related to probability (Duncan-Johnson \& Donchin, 1977; Tueting, Sutton, \& Zubin, 1970), we found no statistically significant difference for signals detected in the two observation intervals $(p=.063)$. Furthermore, the direction of the nonsignificant difference (larger P300 amplitude for signals detected in OI 2) is opposite to the direction predicted on the basis of probability.
P300, signal presence vs. signal absence. Since, in other detection experiments (e.g., K. Squires et al., 1975a), relatively large P300s were obtained to signal absence, it is worthy of notice that, in the present experiment, when the signal was absent P300s were small or absent. The relatively small amplitude of the P300s obtained to signal absence was probably due to the convergence of several factors, all of which tended to make signal absence less salient. First, the instructions specifically made signal presence the target. Subjects were instructed to report the observation interval in which the signal was perceived. Second, the informational properties of signal absence in a two-interval forced-choice procedure are quite different from the informational properties of signal absence in a yes/no (single-interval) procedure. In a yes/no procedure, when the signal is absent, that absence is the sole source of information in the trial, and the subject explicitly reports signal absence. In a two-interval forced-choice procedure, signal absence and signal presence are logically redundant. If the perception of signal presence is clear, signal absence adds no new information. Finally, we have noted in a prior study (Ruchkin \& Sutton, 1978a) that even in a yes/no procedure signal presence and signal absence do not appear to provide equivalent information. P300 is smaller for signal absence than for signal presence even after latency compensation. This smaller amplitude presumably reflects the fact that signal absence is inherently less informational than signal presence.

P300 and equivocation. We have elsewhere commented on the effect of equivocation (unresolved uncertainty) on P300 amplitude (Ruchkin \& Sutton, $1978 b) . P 300$ is generally smaller, the greater the degree of equivocation. In the present study, P300 is larger when the signal is present than when the signal is absent (see Figure 4). P300 is also larger when rated confidence is high than when rated confidence is low. Both of these findings are consistent with the equivocation formulation.

Despite the fact that Lo-intensity signals are more equivocal than $\mathrm{Hi}$-intensity signals, no main effect of signal intensity was found for P300 amplitude. The interaction of location $x$ intensity is statistically significant in the OI 2 recording epoch. In this interaction, the difference in $\mathrm{P} 300$ amplitude in response to signal presence and signal absence is greater in the Hi-intensity condition than in the Lo-intensity condition. This presumably reflects the fact that signal presence and signal absence are more distinguishable at $\mathrm{Hi}$ intensity than at Lo intensity, again in line with the equivocation formulation.

One caveat should be entered with respect to P300 amplitude findings in relation to equivocation. Since conditions that are more equivocal (signal absence, low rated confidence, low signal intensity) would also 
tend to have increased trial-to-trial jitter of P300 latency, without latency compensation it remains possible that smaller mean amplitude is accounted for by increased latency jitter. Elsewhere (Ruchkin \& Sutton, 1978a), we found that the smaller mean amplitude of P300 obtained for signal absence than for signal presence survived latency compensation. However, this does not mean that smaller mean amplitude obtained for other sources of equivocation (or even signal absence in another study) would be maintained if latency compensation were undertaken. Unfortunately, it is our experience that latency compensation cannot be meaningfully used unless signal-to-noise ratios exceed some minimum (at least 2.5). We therefore did not attempt latency compensation in the present study, since P300 is at relatively small amplitude when signals of the intensities used in this experiment are being discriminated from signal absence.

In the present data, it appears that equivocation also influences P300 mean latencies. Even at high confidence, the decision that a Hi-intensity signal has occurred is less uncertain than the decision that a Lo-intensity signal has occurred, and this difference in equivocation is reflected in an earlier P300 for the $\mathrm{Hi}$-intensity signals. It should be noted that random, unimodal trial-to-trial latency jitter (Ruchkin \& Sutton, 1978a) has different effects on the measurement of mean amplitudes as against mean latencies. While greater latency jitter introduces systematic error into mean amplitude measurement (bias toward smaller amplitude), its effect on mean latencies is to introduce random error by increasing variability of measurement. In this sense, mean latency measures are more robust, that is, more resistant to systematic distortion as a result of trial-to-trial latency jitter, than mean amplitude measures.

The shorter latency of P300 for Hi-intensity signals than for Lo-intensity signals presumably reflects the fact that less equivocal decisions can be made more rapidly. Since we were unable to measure P300 latency for low-confidence decisions, we were not able to test whether latency was shorter for highconfidence detections than for low-confidence detections as observed by Kerkhof (1982).

It should be noted that we are not suggesting that P300 is the correlate of the decision process, or that decisions are made at P300 latency. There is ample evidence that the latency of the earlier N2 component is correlated with decision latency (Renault \& Lesevre, 1979; Ritter, Simson, Vaughan, \& Friedman, 1979; Towey, Rist, Hakerem, Ruchkin, \& Sutton, 1980 ), and therefore N2 more probably than P300 is the correlate of the decision. However, P300, which reflects postdecision evaluation, has a latency that is often highly correlated with N2 latency. In the present study we were not able to see and measure N2 reliably, nor was it extracted as a component in the PCVAs. This is probably due to the fact that N2 (in contrast to P300) is a relatively small component, and trial-to-trial latency jitter may wash it out in the average waveform.

Scalp distribution of P300. Up to this point, we have avoided the term "P3b" with respect to our P300 findings, despite the fact that we based the experimental design on reasoning related to the properties of P3b.' The reason for this reticence is that, in the PCVA analyses, when P300 was large it was largest at $\mathrm{Cz}$ (Figure 3) and not at $\mathrm{Pz}$ as would be expected for P3b. The P3b component has generally been found to be maximal at the parietal electrode, both on the basis of the baseline-to-peak measures and also on the basis of PCVA-derived measures. However, there are exceptions reported both for baseline-to-peak measures (Hillyard, Courchesne, Krausz, \& Picton, 1976) and for PCVA-derived measures (K. Squires, Donchin, Herning, \& McCarthy, 1977).

Despite the fact that the main effect of electrodes was not statistically significant in either observation interval, two further checks were undertaken to verify the $\mathrm{Cz}$ maximum for P300 that can be seen in Figure 3. In the four nested conditions that were the only ones to yield large P300s (for high-confidence detections, $\mathrm{Hi}$ and Lo intensity, for signals presented and detected in the OI 1 and in the OI 2 recording epochs), we did $t$ tests comparing the $\mathrm{Cz}$ and $\mathrm{Pz}$ weighting coefficients for the 13 subjects. We also did separate PCVAs on each of these four nested conditions. ${ }^{2}$ Both approaches supported the conclusion that it was a $\mathrm{Cz}$ maximal $\mathrm{P} 300$.

Nevertheless, one must be cautious in dealing with a scalp topography that is based exclusively on PCVA findings and, because of the nature of the overlap, cannot be gleaned from the average waveforms for individual subjects. It is conceivable that the Cz maximal P300 pulled out by the PCVA resulted from the inability of the PCVA to accomplish an exact quantitative separation of $\mathrm{P} 300$ and other overlapping components. While PCVA is capable of extracting information that cannot be directly gleaned from the average waveforms, it is also capable of producing misleading results. Without converging operations, one cannot be sure which has been achieved. For example, different relative latencies of the various underlying components across experimental conditions could create a confound in the PCVA-derived estimates. We dealt with this by doing additional separate PCVAs for nested experimental conditions. However, different relative latencies of the various underlying components across subjects in the same experimental condition, which we could not deal with, could also create a confound in the PCVAderived estimates. The specification of scalp topog- 
raphy would be strengthened if one could confirm it in raw average waveforms under experimental conditions that were devised to reduce temporal overlap among components.

Even if the $\mathrm{Cz}$ maximal scalp distribution for $\mathrm{P} 300$ in the two-interval forced-choice task were convincingly demonstrated, it is not clear at this time what different scalp distributions might imply in terms of differences in functional role. In a prior study, we obtained a $\mathrm{Pz}$ maximal scalp distribution based on PCVA in a yes/no single-interval detection. But Hillyard et al. (1976), using baseline-to-peak measures, reported that a $\mathrm{CZ}$ maximum was obtained in some tasks. While part of the problem arises from the use of different measures in different experiments (baseline-to-peak vs. PCVA-derived measures), this does not appear to account for the variation found. Hillyard et al. (1976) reported fifferences in scalp distribution for different subjects in the same experimental paradigm, as well as differences in average topography (across subjects) as a function of experimental paradigm. As of the present writing, no coherent picture emerges, and further attention to this problem is needed.

\section{Slow-Wave Results}

Although the design of this experiment was based on hypotheses about P300, the Slow-Wave findings are also dealt with in depth. This is done because Slow-Wave data have been rapidly accumulating in

\section{SLOW WAVE}

\section{OII EPOCH}

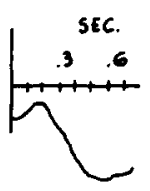

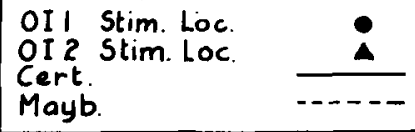
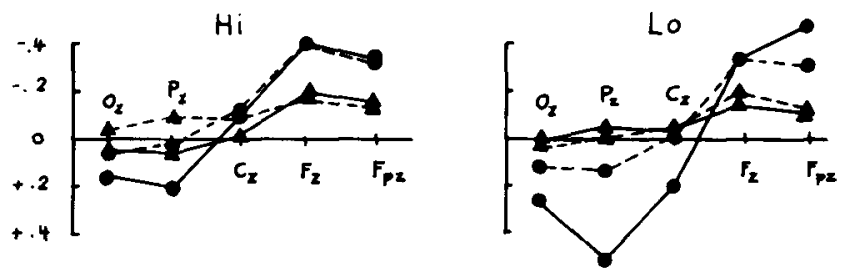

\section{OI 2 EPOCH}
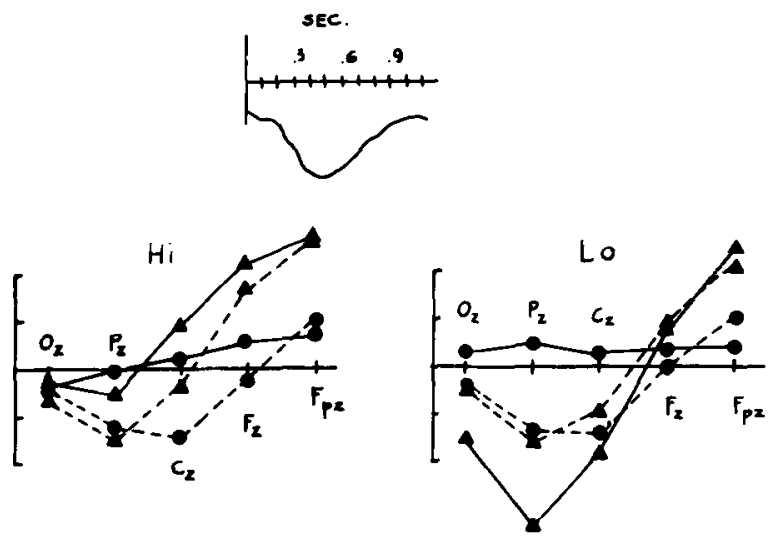

Flgare 5. The Slow-Wave component derived from separate PCVAs of the OI 1 and 012 recording epochs. 
the literature in recent years, and the findings of the current study may broaden our understanding of this component.

A Slow-Wave component was obtained in both the OI 1 and OI 2 recording epochs. The basis waveforms are shown in the upper portion of each panel of Figure 5.

For both recording epochs, Slow Wave reaches peak amplitude at about $500 \mathrm{msec}$. For the OI 2 recordingepoch Slow Wave, return to baseline occurs by about $900 \mathrm{msec}$. For the OI 1 recording epoch, the Slow Wave is still relatively large in amplitude by the end of the epoch $(700 \mathrm{msec})$. A supplementary PCVA spanning both recording epochs indicated that the OI 1 Slow Wave does not get close to baseline until $300 \mathrm{msec}$ after the onset of the OI 2 recording epoch.

For both recording epochs, Slow Wave tends to be positive posteriorly and negative frontally (lower portion of each panel of Figure 5). The main effect of electrodes is statistically significant for both OI 1 $(\mathrm{F}=9.76, \mathrm{p}<.01)$ and $\mathrm{OI} 2(\mathrm{~F}=18.45, \mathrm{p}<.01)$ recording epochs. In the raw averages, some visualization of Slow Wave can be achieved by comparing the basis waveforms in Figure $S$ with the averages for the Pz electrode in Figures 1 and 2.

Slow Wave at Pz. Table 1 shows the $F$ and $p$ values for ANOVA comparisons for Slow Wave at $\mathrm{Pz}$. Slow Wave at $\mathrm{Pz}$ is larger in amplitude (more positive) when the signal is present than when the signal is absent for both recording epochs (Figure 6). In the OI 1 recording epoch, $\mathrm{Pz}$ Slow Wave is larger in amplitude for high-confidence detections (C) than for low-confidence detections (M). There is also a clear trend for $\mathrm{Pz}$ Slow Wave to be larger at Lo intensity than at $\mathrm{Hi}$ intensity for both recording epochs.

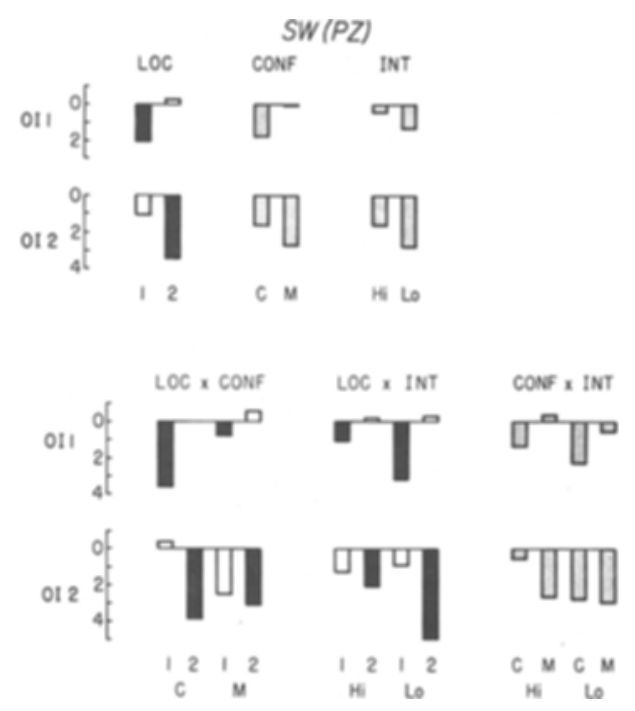

Figure 6. Across-gubject means for Slow Wave at Pz. All codes are as in Figure 4.

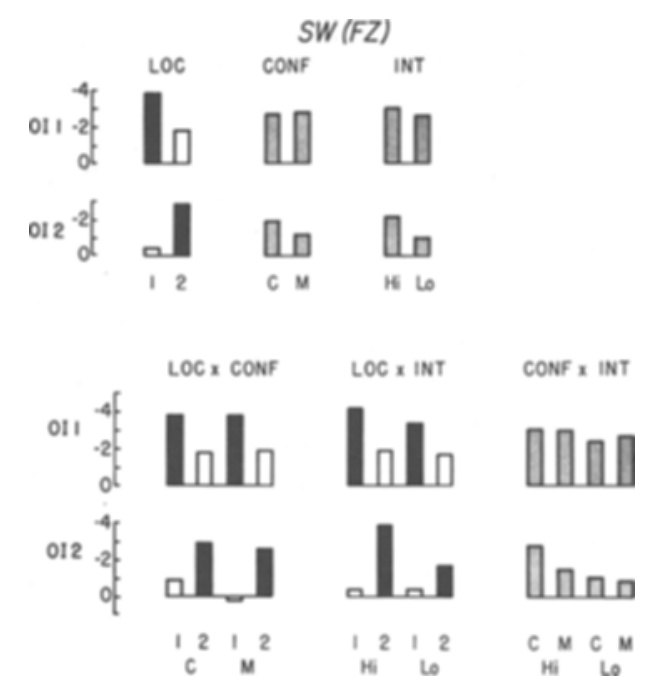

Figure 7. Across-subject means for Slow Wave at Fz. All codes are as in Figure 4.

All three two-variable interactions for $\mathrm{Pz}$ Slow Wave-location $x$ confidence, location $x$ intensity, and confidence $x$ intensity (discussed in greater detail below)-were either statistically significant or showed a clear trend in the OI 2 recording epoch. Both location $x$ confidence and location $x$ intensity also showed trends in the same direction in the OI 1 recording epoch.

(1) Location $\times$ confidence-In the OI 2 recording epoch, for high-confidence $(C)$ detections, signal present yields larger $\mathrm{Pz}$ Slow Wave than signal absent, whereas for low-confidence (M) detections, the difference between signal present and signal absent is small. The trend in the ol 1 recording epoch is in the same direction.

(2) Location $x$ intensity-In the OI 2 recording epoch, at Lo intensity, $\mathrm{Pz}$ Slow Wave is larger for signal present than for signal absent, whereas at $\mathrm{Hi}$ intensity, the difference between signal present and signal absent is small. The trend in the OI 1 recording epoch is in the same direction.

(3) Confidence $x$ intensity-There is a trend in the OI 2 recording epoch for $\mathrm{Pz}$ Slow Wave to have larger amplitude for Lo intensity than for Hi intensity when detections are made at high confidence $(C)$, whereas there is little difference between LO and Hi intensity when detections are made at low confidence (M). No such trend was found for the OI 1 recording epoch.

Signal location, confidence, and intensity interact in both recording epochs. The details of this interaction are complex, and they are dealt with in the $\mathbf{P z}$ section of Slow-Wave Discussion below.

Slow Wave at $\mathrm{Pz}$ was significantly larger $(\mathrm{F}=$ 12.61, $\mathrm{p}=.004$ ) in the OI 2 recording epoch than in the OI 1 recording epoch.

Slow Wave at Fz. Table 1 shows the $F$ and $p$ values for ANOVA comparisons for Slow Wave at 
Fz. For both recording epochs, Fz Slow Wave is more negative for signal present than for signal absent (Figure 7). For the OI 2 recording epoch, Fz Slow Wave is larger (more negative) for Hi-intensity signals than for Lo-intensity signals.

There is a trend in the OI 2 recording epoch for the difference between signal presence and signal absence to be larger at $\mathrm{Hi}$ intensity than at Lo intensity.

There is a trend $(F=5.32, p=.04)$ for Slow Wave at $\mathrm{Fz}$ to be larger (more negative) in the OI 1 recording epoch than in the OI 2 recording epoch.

\section{Slow-Wave Discussion}

Pz Slow Wave. We have noted elsewhere (Ruchkin, Sutton, Kietzman, \& Silver, 1980; Ruchkin \& Sutton, in press) that the effect of equivocation on $\mathrm{Pz}$ Slow Wave tends to be opposite to its effect on P300, with larger $\mathrm{Pz}$ Slow Wave associated with greater equivocation. In the current experiment, (1) Pz Slow Wave was larger in both recording epochs for signal present than for signal absent; (2) in the OI 1 recording epoch, it was larger for high-confidence detections than for low-confidence detections; and (3) in both recording epochs, it tended to be larger for Lointensity signals than for Hi-intensity signals (Figure 6). Of these three main effects, only the association or larger $\mathrm{Pz}$ Slow Wave with Lo-intensity signals goes along with the Equivocation formulation for Slow Wave.

In a prior study, the relationship of Pz Slow Wave to equivocation was an empirical finding and a derivative formulation (Ruchkin, Sutton, Kietzman, \& Silver, 1980). Our more general formulation for Slow Wave was that its amplitude indexed the degree of processing effort. Stimuli that were more equivocal could be interpreted as requiring greater processing effort. The present findings can be better understood if we broaden the concept of processing effort. It is suggested here that when there is redundant information, $\mathrm{Pz}$ Slow Wave will be elicited by those stimuli which not only require processing effort, but also provide the best available source of information to make such processing feasible.

Bearing this expanded formulation in mind, we will focus on the three-variable interactions for $\mathrm{Pz}$ Slow Wave. In Figure 8, the three-variable interactions are shown for P300 as well as for Pz Slow Wave. Although these interactions for $P 300$ were not statistically significant, ${ }^{3}$ they are useful in developing a more coherent interpretation of the findings for Pz Slow Wave.

In examining Figure 8, it might be remembered that each vertical column of bars represents the same set of trials. Thus, for example, the leftmost ( $\mathrm{HiCl}$ ) bars show that in the same set of trials P300 is large in OI 1 for signal presence and small in OI 2 for

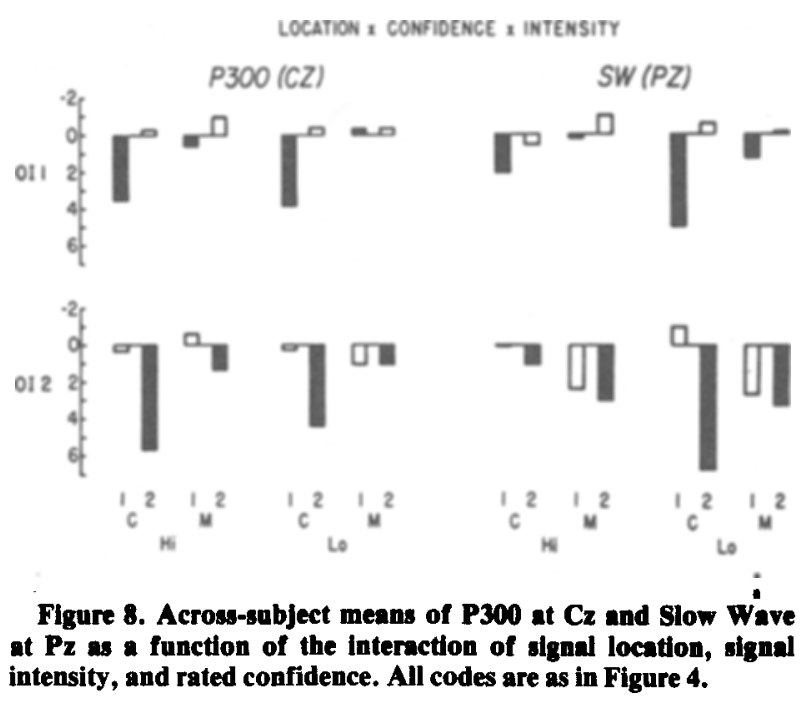

signal absence. We should also bear in mind that P300 amplitude is believed to index an initial evaluation, whereas Slow-Wave amplitude is believed to index processing effort whether or not an initial evaluation has been made.

In general, the findings for $\mathrm{P} 300$ suggest that at high confidence (C), for both $\mathrm{Hi}$ and Lo intensity signals, evaluation and therefore a decision with respect to the trial is made in the recording epoch corresponding to the interval in which the signal is presented (large-amplitude P300s). In contrast, the Slow-Wave findings at high confidence (C) suggest that processing effort, while also linked to signal presence, is large only at Lo intensity (OI 1 recording epoch, LoC1; OI 2 recording epoch, LoC2). For high-confidence detections, little processing effort may be necessary for the Hi-intensity signals, whereas processing effort is necessary for the Lo-intensity signals, but it is most feasible to make the effort when the signal is present.

For Lo-confidence decisions (M), the consistently small amplitude of $\mathrm{P} 300$ suggests that a decision is probably not made in either recording epoch. Slow Wave is also small for both signal presence and signal absence in the OI 1 recording epoch, but in the OI 2 recording epoch, Slow Wave is of intermediate and almost equal amplitude for signal presence and signal absence. These findings are interpreted to mean that for those trials in which confidence in the decision is reported to be low, little processing effort was made in OI 1 since there was still upcoming information in OI 2 which might clarify where the signal is located. However, the subject remained unsure in the OI 2 recording epoch, but this was the last opportunity in the trial to extract information. The subject therefore made some effort to process, and here even signal absence was processed, since there was no further source of information. 
In summary, we return to our broadened conceptualization of the relationship between equivocation and processing effort. We suggested that to elicit $\mathrm{Pz}$ Slow Wave, stimuli must require processing effort, and must also provide the best available information to make such effort feasible. Thus, we find relatively little $\mathrm{Pz}$ Slow Wave for high-confidence detections of $\mathrm{Hi}$-intensity signals, since little processing effort is required; and we also find little $\mathrm{Pz}$ Slow Wave in the OI 1 recording epoch at low confidence, since not enough information has yet been received to make such processing feasible. However, for low-confidence detections, there is somewhat more information by the OI 2 recording epoch, since information from both observation intervals is now available, and with this somewhat increased feasibility of processing, we find an intermediate level of $\mathrm{Pz}$ Slow Wave. Finally, when Lo-intensity stimuli-which require processing effort-are presented and detected with confidence, thus making the processing quite feasible, we find the largest amplitude of $\mathrm{Pz}$ Slow Wave.

The above considerations suggest that various sources of equivocation do not appear to act independently in determining the amplitude of $\mathrm{Pz}$ Slow Wave. Rather, it is the specific way various sources of equivocation interact in a given task that determines the degree of processing effort which in turn determines the amplitude of $\mathrm{Pz}$ Slow Wave.

Fz Slow Wave. The differences in the effect of experimental variables at $\mathrm{Fz}$ and $\mathrm{Pz}$ do not seem to be attributable to a simple reversal in polarity. While such an explanation would hold for the effect of signal location, that is, when the signal is present there is more positivity at $\mathrm{Pz}$ (Figure 6) and more negativity at $\mathrm{Fz}$ (Figure 7), the effects of signal intensity in the OI 2 recording epoch do not follow this pattern (Figures 6 and 7). At $\mathrm{Fz}, \mathrm{Hi}$ intensity is associated with more negativity, whereas at $\mathrm{Pz}$ there is a trend for Lo intensity to be associated with more positivity.

The PCVA yielded only one Slow Wave component, despite some differences in findings at $\mathrm{Pz}$ and $\mathrm{Fz}$ (Figures 5, 6, and 7). Fitzgerald and Picton (1981), using baseline-to-peak measures, reported different findings in relation to experimental conditions for Slow Wave at $\mathrm{Pz}$ and $\mathrm{Fz}$. They suggested that the parietal Slow Wave and the frontal Slow Wave might be different components. It is possible that our PCVAs were not able to separate what were, in fact, two different components because they have the same time course (Friedman, Note 1). Fitzgerald and Picton (1981) noted that Slow Wave at Fz was more sensitive to information load than was Slow Wave at $\mathrm{Pz}$. The present experiment was not designed to deal with this question, and it would be difficult to do so post hoc.

\section{Other Components}

Two additional PCVA-derived components, the slow negative shift component and the late negative component, are presented minimally because the findings do not elucidate the question to which this experiment was addressed and these components are not well understood in their own right.

The slow negative-shift component is a sustained negativity which tends to be slightly more negative for the initial portion of both recording epochs. It is at maximum amplitude at $\mathbf{C z}$ and $\mathbf{P z}$. Its behavior in relation to experimental variables resembles that of $\mathrm{Pz}$ Slow Wave except that it is more negative when $\mathrm{Pz}$ Slow Wave is more positive. The slow negativeshift component found in the current experiment appears to be quite similar to the sustained negativity reported by Picton et al. (1978) in a fourinterval forced-choice paradigm.

The late negative component was obtained only in the PCVA of the OI 2 recording epoch. It is most negative at the frontal electrodes and slightly positive at the occipital electrode. No relationship was found between this component and any experimental variables. The lack of relationship to experimental variables and its presence only in the OI 2 recording epoch suggest that the late negative component may represent a nonspecific preparation of the subject to report the decision.

\section{TWO-INTERVAL FORCED-CHOICE DECISION STRATEGY}

If we focus on the P300 findings, and if we assume, as is widely accepted, that P300 reflects postdecision evaluation, fairly clear inferences may be made on how subjects make decisions in a twointerval forced-choice detection task. In terms of the alternative possible findings that were outlined in the introduction, two of the four can be rejected. P300 is not always largest in the first recording epoch (1), nor is it always largest in the second recording epoch (2). One of the alternatives (4) is what is found for high-confidence detections; that is, P300 is largest in the recording epoch in which the signal is presented. The remaining alternative (3) is what is found for low-confidence detections; that is, P300 is small or absent in both recording epochs.

With respect to the issue raised by Treisman and Leshowitz (1969) as to whether the subject makes serial independent detections or is involved in some comparison operation, the P300 findings suggest that different strategies are used at high and low confidence.

For high-confidence detections, the subject appears to use a strategy involving serial independent detection. In both recording epochs, P300 is large when the signal is present and negligible when the 
signal is absent. However, the latency data suggest that decisions that the signal is present in the second observation interval are not entirely independent of the information provided by the absence of the signal in the first observation interval. Latency of P300 is 100 msec shorter in the second recording epoch than in the first recording epoch. The latency difference suggests that signal absence in the first observation interval is not ignored: Presumably, signal absence in the first observation interval is noted at some level and makes possible a more rapid decision that the signal is present in the second observation interval. This inference is consistent with behavioral data which show that two-interval forcedchoice detection yields a more sensitive $\mathrm{d}^{\prime}$ than yes/ no detection (Schulman \& Mitchell, 1966). In our study, corresponding amplitude findings were not obtained. P300 is not significantly larger in the OI 2 recording epoch than in the OI 1 recording epoch.

At low confidence, the P300 findings do not suggest a serial detection strategy. For both recording epochs, P300 is small or negligible in amplitude for low-confidence detections, whether the signal is present or absent (Figure 4). While there is no direct evidence on the subject's decision strategy when at low confidence, the P300 data imply a deferral of decision, which suggests that the subject may utilize information from both observation intervals and make some kind of comparison of the two percepts.

We had no a priori predictions with respect to what other components would tell us with respect to the nature of the subject's strategy in a twointerval forced-choice task. Rather, for Pz Slow Wave, we inferred the subject's strategy in an attempt to broaden our understanding of its functional role. The most consistent finding for all components except the late negative component was that signal presence yielded larger amplitude than signal absence. This finding suggests that the various aspects of processing, which are presumably reflected by the different components, are all affected by the properties of the task which resulted in focusing primarily on signal presence.

\section{REFERENCE NOTE}

1. Friedman, D. P300 and slow wave: The effects of quartile revisited. Paper presented at the Society for Psychophysiological Research meeting, Washington, D.C., October 1981.

\section{REFERENCES}

Barkett, C., Halliday, A. M., Halliday, E., \& RudolF, N. DE M. The later components of the somatosensory evoked potential and the $\mathrm{P} 300$ in a threshold detection task. In J. E. Desmedt (Ed.), Cognitive components in cerebral eventrelated potentials and selective attention. Progress in clinical neurophysiology. Basel: Karger, 1979.

Campbele, K. B., Courchesne, E., Picton, T. W., \& SQuires, K. C. Evoked potential correlates of human information processing. Biological Psychology, 1979, 8, 45-68.
Duncan-Johnson, C. C. P300 latency: A new metric of information processing. Psychophysiology, 1981, 18, 207-215.

Duncan-Johnson, C. C., \& Donchin, E. On quantifying surprise: The variation of event-related potentials with subjective probability. Psychophysiology, 1977, 14, 456-467.

Egan, J. P., Greenberg, G. Z., \& Schulman, A. I. Interval of time uncertainty in auditory detection. In J. A. Swets (Ed.), Signal detection and recognition by human observers. New York: Wiley, 1964.

Emmerich, D. S., Gray, J. L., Watson, C. S., \& Tanis, D. C. Response latency, confidence, and ROCs in auditory signal detection. Perception \& Psychophysics, 1972, 11, 65-72.

Fitzgerald, P. G., \& Picton, T. W. Temporal and sequential probability in evoked potential studies. Canadian Journal of Psychology, 1981, 35, 188-200.

Friedman, D., Simson, R., RitTer, W., \& Rapin, 1. The late positive component (P300) and information processing in sentences. Electroencephalography and Clinical Neurophysiology, $-1975,38,255-262$.

Friedman, D., Vaughan, H. G., Jr., \& Erlenmeyer-Kimling, L. Stimulus and response related components of the late positive complex in visual discrimination tasks. Electroencephalography and Clinical Neurophysiology, 1978, 45, 319-330.

Hillyard, S. A., Courchesne, E., Krausz, H. I., \& Picton, T. W. Scalp topography of the P3 wave in different auditory decision tasks. In W. C. McCallum \& J. R. Knott (Eds.), The responsive brain. Bristol: Wright, 1976.

Hillyard, S. A., Squires, K., Bauer, J. W., \& Lindsay, P. H. Evoked potential correlates of auditory signal detection. Science, $1971,172,1357-1360$.

Jennings, J. R., \& Woods, C. The e-adjustment for repeatedmeasures analysis of variance. Psychophysiology, 1976, 13, 277-278.

Johnson, R., JR., \& Donchin, E. Sequential expectancies and decision making in a changing environment: An electrophysiological approach. Psychophysiology, 1982, 19, 183-200.

KERKHor, G. A. Event-related potentials and auditory signal detection: Their diurnal variation for morning-type and eveningtype subjects. Psychophysiology, 1982, 19, 94-103.

Picton, T. W., Campbell, K. B., Baribeau-Braun, J., \& Proulx, G. B. The neurophysiology of human attention: A tutorial review. In J. Requin (Ed.), Attention and performance VII. Hillsdale, N. J: Erlbaum, 1978.

REnautT, B., \& Lesevre, N. A trial by trial study of the visual omission response in reaction time situations. In D. Lehmann \& E. Callaway (Eds.), Human evoked potentials: Applications and problems. New York: Plenum, 1979.

Ritter, W., Simson, R., Vaughan, H. G., JR., \& Friedman, D. $A$ brain event related to the making of a sensory discrimination. Science, 1979, 203, 1358-1361.

RitTer, W., \& Vaughan, H. G., JR. Averaged evoked responses in vigilance and discrimination: A reassessment. Science, 1969, 164, 326-328.

Ruchkin, D. S., Mungon, R., Sutton, S., \& Kietzuan, M. L. P300 and Slow Wave in a message consisting of two events. Psychophysiology, in press.

Ruchkin, D. S., \& SuTton, S. Emitted P300 potentials and temporal uncertainty. Electroencephalography and Clinical Neurophysiology, 1978, 45, 268-277. (a)

Ruchkin, D. S., \& Sutron, S. Equivocation and P300 amplitude. In D. Otto (Ed.), Multidisciplinary perspectives in event-related brain potential research. Washington, D.C: U.S. Government Printing Office, 1978. (b)

Ruchkin, D. S., \& Sutron, S. P300 and slow wave-Association and dissociation. In A. W. K. Gaillard \& W. Ritter (Eds.), Tutorials in event related potential research: Endogenous components. Amsterdam: North Holland, in press.

Ruchkin, D. S., Sutton, S., Kietzman, M. L., \& Silver, K. Slow Wave and P300 in signal detection. Electroencephalography and Clinical Neurophysiology, 1980, 50, 35-47.

Ruchrin, D. S., Sutron, S., \& StegA, M. Emitted P300 and 
slow wave potentials in guessing and detection tasks. Electroencephalography and Clinical Neurophysiology, 1980, 49, 1-14.

Schulman, A. I., \& Mitchell, R. R. Operating characteristics from yes/no and forced-choice procedures. Journal of the Acoustical Society of America, 1966, 40, 473-477.

Shelburne, S. A., Jr. Visual evoked responses to word and nonsense syllable stimuli. Electroencephalography and Clinical Neurophysiology, 1972, 32, 17-25.

Squires, K., Donchin, E., Herning, R. T., \& McCarthy, G. On the influence of task relevance and stimulus probability on event-related potential components. Electroencephalography and Clinical Neurophysiology, 1977, 42, 1-14.

Squires, K., Hillyard, S. A., \& Lindsay, P. H. Cortical potentials evoked by confirming and disconfirming feedback following an auditory discrimination. Perception \& Psychophysics, 1973, 13, 25-31.

Squires, K., Seuires, N., \& Hillyard, S. A. Decision related cortical potentials during an auditory signal-detection task with cued observation intervals. Journal of Experimental Psychology: Human Perception and Performance, 1975, 1, 268-279. (a)

Squires, K., Squires, N., \& Hillyard, S. A. Vertex evoked potentials in a rating-scale detection task: Relation to signal probability. Behavioral Biology, 1975, 13, 21-34. (b)

Squtres, N., Squires, K., \& Hillyard, S. A. Two varieties of long-latency positive waves evoked by unpredictable stimuli in man. Electroencephalography and Clinical Neurophysiology, 1975, 38, 387-401.

Sutton, S., Braren, M., Zubin, J., \& John, E. R. Evoked potential correlates of stimulus uncertainty. Science, 1965, 150, 1187-1188.

Sutton, S., \& Ruchkin, D. S. The late positive complexAdvances and new problems. In R. Karrer, J. Cohen, \& P. Tueting (Eds.), Brain and information: Event-related potentials. New York: New York Academy of Sciences, in press.

Sutron, S., Tuetina, P., Zubin, J., \& John, E. R. Information delivery and the sensory evoked potential. Science, 1967, 155, 1436-1439.

Towey, J., Rist, F., Hakerem, G., Ruchinin, D. S., \& Sutron, S. N250 latency and decision time. Bulletin of the Psychonomic Society, 1980, 15, 365-368.

Treisman, M., \& Leshowitz, B. The effects of duration, area, and background intensity of the visual intensity difference threshold given by the forced-choice procedure: Derivations from a statistical decision model for sensory discrimination. Perception \& Psychophysics, 1969, 6, 281-295.

Tueting, P., SutTon, S., \& Zubin, J. Quantitative evoked potential correlates of the probability of events. Psychophysiology, 1970, 7, 385-394.

Wilkinson, R. T., \& Seales, D. M. Event related potentials in signal detection. Biological Psychology, 1978, 7, 13-28.

\section{NOTES}

1. Terminological problems in this area are quite severe and cannot be resolved by fiat since they not only involve inconsistent usage in the literature, but also involve conceptual and empirical issues. For a discussion of these issues, see Sutton and Ruchkin (in press). The term P3b was introduced by N. Squires, K. Squires, and Hillyard (1975) in order to distinguish P3b from a shorter latency, centrofrontal maximum component (P3a), which had a different functional role. It has generally been assumed, and this assumption is shared by the present authors, that P3b corresponds to the classical late positive component first reported by Sutton et al. (1965). This late positive component, or LPC, later became known as P3 or P300. However, because earlier studies did not report scalp distribution, and because the problem of temporal and spatial overlap of several components in the same latency region has only come to the fore in recent years, it is no longer possible to assume that all the functional properties attributed to the late positive component (LPC, late positive complex, P3, $\mathrm{P} 3 \mathrm{~b}$, or $\mathrm{P} 300$ ) are, in fact, exclusively or primarily the properties of one component. In the current paper, the term P300 is used in order to avoid specifying exactly which component is being referred to (as this is not always possible), whereas the term P3b is used at this point to refer explicitly to the classical component, whose functional role has been described by such constructs as uncertainty reduction and expectancy.

2. We are indebted to Nancy Squires for suggesting this approach. Friedman, Vaughan, and Erlenmeyer-Kimling (1978) have used this approach in the past to clarify the relationship among PCVA-derived components.

3. The three-variable interactions for $\mathbf{P} 300$ at $\mathbf{C z}$ are not statistically significant because the same pattern is obtained at $\mathrm{Hi}$ and Lo intensity. The P300 data are plotted separately for $\mathrm{Hi}$ and Lo intensity in Figure 8 only in order to facilitate comparison with Pz Slow-Wave data. For P300, across both intensities, the interaction of location with confidence is statistically significant for both OI 1 and OI 2 (Figure 4 and Table 1), and it is this interaction which is used in the reasoning in this section.

(Manuscript received April 6, 1982; revision accepted for publication July 28,1982 .) 